\title{
Los desafíos metodológicos en el estudio de las alianzas electorales subnacionales
}

\author{
Methodological challenges in the study \\ of subnational electoral coalitions
}

\author{
doi: https://doi.org/10.32870/ \\ eees.v27i77.7096
}

\author{
Orlando Espinosa Santiago \\ Ignacio Daniel Torres Rodríguez*• \\ Ulises Cruz Valencia»••
}

\section{Resumen}

Este artículo parte de las tensiones metodológicas registradas en la literatura reciente sobre alianzas electorales entre estudios cuantitativos y los registros empíricos de elecciones donde no se cumplen las expectativas teóricas. Buscando encontrar las razones de esta anomalía, se propone identificar los factores que inhiben el efecto de las variables independientes -los llamados mecanismos causales-, a partir de estudios de caso. En este sentido, el artículo expone el giro metodológico al respecto, previa revisión de las limitaciones de los estudios cuantitativos y la necesidad de complementarlos desde una aproximación cualitativa. Mediante un ejemplo de selección intencionada de observaciones, se visualiza la riqueza del ejercicio metodológico para posteriores estudios. Se concluye que los casos típicos, atípicos, de contraste típico-atípico y de máxima variación pueden afinar el conocimiento sobre los mecanismos y condiciones que posibilitan las alianzas electorales.

Palabras clave: metodología, alianzas electorales, elecciones subnacionales, estudios de caso, selección intencionada.

\begin{abstract}
This article is based on the methodological tensions recorded in the recent literature on pre-electoral coalitions between quantitative studies and the empirical records of elections where theoretical expectations are not met. In order to find the reasons for this anomaly, it is proposed to identify the factors that inhibit the effect of independent variables -the so-called causal mechanisms, based on case studies. In this sense, the article exposes the methodological turn in this regard, after reviewing the limitations of quantitative studies and the need to complement them from a qualitative approach. Through an example of intentional selection of observations, the richness of the methodological exercise for subsequent studies is visualized. It is concluded that the typical, atypical, typical-atypical contrast and maximum variation cases can refine the knowledge about the mechanisms and conditions that enable pre-electoral coalitions.
\end{abstract}

Keywords: Methodology, pre-electoral coalitions, subnational elections, case studies, intentional selection.

\footnotetext{
- Profesor-Investigador del Instituto de Ciencias de Gobierno y Desarrollo Estratégico (ICGDE) de la Benemérita Universidad Autónoma de Puebla (BUAP), México. ORCID: https://orcid.org/0000-0003-4260-0365 orlando.espinosa@correo.buap.mx

- Investigador del Instituto de Ciencias de Gobierno y Desarrollo Estratégico (ICGDE) de la Benemérita Universidad Autónoma de Puebla (BUAP), México. ORCID: https://orcid.org/0000-0003-4205-039I ignacio.torres@correo.buap.mx

- Profesor-Investigador del Departamento de Comunicación de la Universidad Iberoamericana sede Santa Fe, Ciudad de México.orCID:https://orcid.org/0000-000I-9794-70I I ulises.cruz@ibero.mx Fecha de recepción: 28 de marzo de 2019. Fecha de aceptación: 09 de septiembre de 2019.
} 
Introducción

Desde la década de los noventa en México, las alianzas electorales -definidas como el "conjunto de partidos que no compiten de manera independiente en una elección, ya sea porque acuerdan coordinar sus campañas de manera pública, lanzar candidatos o planillas conjuntas, o gobernar coaligados después de la elección" (Golder, 2005, p. 652) - ${ }^{1}$ se han constituido en una práctica recurrente de los partidos políticos para maximizar sus probabilidades de victorias electorales.

En un inicio, las alianzas concretadas por la oposición se justificaron con el objetivo de vencer al Partido Revolucionario Institucional (PRI) (González, 2017; García; 2017), dada la hegemonía con que mantenía el poder en los tres niveles de gobierno en pleno proceso de transición política. No obstante, conforme las elecciones se volvieron más competitivas, las alianzas fueron cada vez más replicadas hasta volverse una de las estrategias más utilizadas por los partidos políticos en campaña. Hasta 2018, el PRI era uno de sus máximos exponentes (Reynoso, 2011a; Espinosa, 2013).

Académicamente, el fenómeno se ha presentado incrementalmente como un observable que los estudiosos de las ciencias sociales han tratado de explicar. No obstante, en su mayoría lo han hecho desde el análisis de muestras amplias enfocadas en variables, más que en casos particulares. Si bien en los estudios previos los porcentajes de correlación entre variables se muestran bastante significativos, hay casos en que la teoría no ofrece respuestas satisfactorias

I.También pueden ser entendidas como "un grupo de partidos que coordinan sus fuerzas detrás de un candidato (o candidatos) común con el objetivo de obtener un desempeño electoral cuantitativa y cualitativamente superior del que obtendrían compitiendo individualmente" (Reynoso, 20 I0, p. I I4); o bien, como "estrategias de acción colectiva entre partidos que implican un proceso de negociación que determina los costos y beneficios que obtiene cada una de las partes y que están hipotéticamente dispuestos a pagar por no competir de manera independiente" (Méndez, 20I2, p. I5I). 
y en los que resulta menester indagar qué condiciones son las que inhiben o bloquean los efectos esperados.

La investigación reciente sobre alianzas electorales en el ámbito de las elecciones de gobernador en el país ha develado este tipo de paradojas entre los efectos esperados de las variables y lo empíricamente observado. Reynoso y Espinosa (2017) han confirmado que las alianzas en Tlaxcala (en las elecciones de 2010) y el Estado de México (en 2011) escapan a los argumentos de la teoría desarrollada tanto por los estudios que analizan cuantitativamente los determinantes de las alianzas electorales (Reynoso, 2010 y 2011a) como por los estudios de caso cualitativos (Reynoso y Espinosa, 2017). Esto sugiere que hay elementos o factores en esos estados que tienen mayor poder explicativo que los arrojados por la literatura previa, sobre todo si se consideran características propias del antiguo sistema de partido hegemónico priista (Reynoso y Espinosa, 2017, p. 411).

La estrategia ampliamente recomendada en estas situaciones por la literatura metodológica es complementar los métodos cuantitativos y cualitativos, sea para combinar muestras de $\mathrm{N}$ grande (de muchas observaciones) y $\mathrm{N}$ pequeña (de pocas observaciones) en una misma investigación (Bäck y Dumont, 2007), o para integrar métodos cuantitativos y cualitativos (Fearon y Laitin, 2008; Goertz y Mahoney, 2012); el propósito final es mostrar los mecanismos causales ${ }^{2}$ de las regresiones estadísticas para constatar si los argumentos que las sustentan son plausibles en contextos específicos.

A su vez, considerados como "ejemplos de una clase de eventos" (George y Bennett, 2005, p. 17), los casos remiten a la generación de teoría o conocimiento genérico con

2. Un mecanismo causal es aquel que expresa una conexión plausible entre $x$ y $y$, que ocurre cuando se conjunta conocimiento general del mundo con conocimiento empírico sobre cómo $x$ y y se interrelacionan, y no sólo sobre cómo varían (Gerring, 2004, p. 348).

Teoría y Debate 8 No.77 
respecto a las causas de similitud o diferencia entre ellos. Dependiendo de los criterios de selección, estos pueden guiar un trabajo para obtener explicaciones diferentes sobre algo en particular.

Por ello, este artículo muestra una serie de criterios de selección de casos, pensados para complementar la teoría sobre las alianzas electorales en la mayor medida posible. Como ha sido subrayado en la tradición cualitativa, ${ }^{3}$ los estudios de caso aportan validez conceptual y procedimientos fuertes para extender hipótesis con mayor profundidad sobre la conexión que sostienen las variables independientes y dependientes, y finalmente, muestran una complejidad causal que muchas veces los estudios cuantitativos no permiten asimilar (George y Bennett, 2005, p. 19).

El ejercicio metodológico vale la pena en casos como los de las alianzas electorales en los estados mexicanos donde el número de las mismas ha descendido considerablemente de un proceso a otro, a la par que se ha evidenciado un súbito incremento en la competitividad electoral. Los casos seleccionados podrían evidenciar pautas sobre las coaliciones en general y sobre el subgrupo de las alianzas entre el Partido Acción Nacional (PAN) y el Partido de la Revolución Democrática (PRD), antes considerados como de oposición, así como generar hipótesis nuevas para ser probadas en muestras más amplias.

Además, dada la profundidad e implicaciones de los elementos aquí descritos, resulta necesario realizar investigación cualitativa y trabajo de campo para desentrañar esos elementos que escapan al enfoque cuantitativo.

De esta forma, a lo largo de este artículo, primero, se desarrolla un apartado donde se enfatiza la tensión cre-

3. Denzin y Lincoln (citados en Newman y Benz, 1998, p. 16) plantean que la investigación cualitativa posee un enfoque nutrido de múltiples métodos e incluye un acercamiento interpretativo y natural de un determinado objeto de estudio. Estudia fenómenos en sus condiciones naturales, $y$ pretende brindarles sentido $o$ interpretación en términos de los significados que la gente les atribuye.

\section{2}


ciente entre el enfoque centrado en variables y el enfoque centrado en casos para el estudio de las alianzas electorales. En un segundo apartado, se evidencian las limitaciones de los estudios cuantitativos en la materia. En el tercero, se revisa el incipiente desarrollo de los estudios cualitativos. En el cuarto, se reitera la necesidad de ampliar la aproximación cualitativa, y en el quinto se realiza una propuesta de casos relevantes teóricamente seleccionados. El texto cierra con un apartado de conclusiones.

\section{La tensión creciente de los estudios sobre alianzas electorales}

Para el caso mexicano, Reynoso (2010 y 2011a), Méndez (2012), Miño (2014) y Devoto y Olmeda (2017) dan cuenta de que las variables de mayor peso contrastadas para explicar la formación de las alianzas electorales son la competitividad, el pragmatismo, la normatividad y la dimensión multinivel de las estrategias partidistas. Las dos primeras, en su acepción general, son factores que condicionan sustantivamente la formación de alianzas, por lo que tienen mayor capacidad explicativa.

De esta manera, las manifestaciones empíricas del fenómeno y los hallazgos de las investigaciones apuntan a las alianzas como una estrategia electoral dominante (Reynoso, 2011a). Esto hace que la atención de los investigadores del fenómeno se centre en explicaciones causales de la formación de las alianzas más que en conocer cuáles son las condiciones mediadoras para formar una alianza.

Es así que, aunque de manera general la literatura pone en evidencia que diferentes hipótesis en torno a la formación de las alianzas han sido probadas, aún es posible encontrar ciertos casos donde las expectativas teóricas no se cumplen, o bien, casos donde los resultados no tienen una explicación plausible del por qué suceden como suceden. 
Se sabe que algunas excepciones de estudios han emergido. Tal es el caso del recientemente realizado por Reynoso y Espinosa (2017), donde a partir de un tratamiento cualitativo los autores buscaron profundizar en las características que distinguen el fenómeno de las alianzas electorales PAN-PRD en comicios para gobernador en México. Los autores observaron que la reducción de alianzas y la nula continuidad de las exitosas es algo que todavía no encuentra explicación en la literatura, es decir, si el primordial objetivo de las alianzas es incrementar las probabilidades de triunfo electoral (Reynoso, 2011a), surge la duda de por qué las alianzas se reducen y, sobre todo, por qué pueden estar ausentes si se presentan las condiciones enunciadas por la teoría para su emergencia.

Sobre la expectativa teórica general de los textos cuantitativos sobre el caso mexicano de las alianzas electorales, ${ }^{4}$ para Reynoso (2011a), por ejemplo, "cuanto mayor sea la volatilidad y menor el margen de victoria en una elección, más probable será que en la elección siguiente se presente una alianza electoral" (Reynoso, 2011a, p. 15), es decir, si existe un ajustado margen de victoria en una elección, los partidos tenderán a formar coaliciones en búsqueda del triunfo, y por el contrario, si se registran márgenes más amplios la probabilidad de formar alianzas disminuye; asimismo, el registro de alianzas en contienda provocará que la oposición replique la estrategia.

Para Miño (2014), de existir un Gobierno dividido vertical, hay mayores probabilidades de formar una alianza divergente; en otras palabras, cuando coincide el partido que

4. Para Méndez (20I2), además de la competitividad, factores como la ideología y la normatividad tienen fuerte incidencia en la formación de alianzas. Ahora bien, debido a que los datos usados en este estudio consideran las elecciones para cargos de diputados federales, gobernadores y presidentes de la república, eso limita la comparación más consistente a los estudios de Reynoso (20 I la) y Miño (20 I4), quienes exclusivamente analizan los casos de alianzas para elecciones de gobernador.

\section{4}


gobierna en el ámbito federal con el partido del gobernador de un estado no se presentará una alianza divergente.

Con el propósito de mostrar la tensión entre estas expectativas teóricas sobre el caso mexicano de las alianzas electorales y lo registrado empíricamente en torno al fenómeno, a continuación se presentan algunos ejemplos de dichos contrastes.

Con respecto al proceso electoral para gobernador en Aguascalientes en 2016, la elección anterior, en 2010, se caracterizó por contar con una alianza en la contienda y un índice de competitividad electoral ${ }^{5}$ de $5.21 \%$. Siguiendo a Reynoso (2011a), para 2016 lo que se debió haber observado sería una reducción en el margen de victoria y un incremento en el número de alianzas. Sin embargo, esto no ocurrió, ya que se mantuvo una alianza, y la diferencia entre el primer y el segundo lugar disminuyó a 2.92\%. Algo similar sucedió en Durango, donde en la elección de 2010 el margen de victoria fue de $1.9 \%$ y también se registró una sola alianza, mientras que para la elección de 2016 el margen de victoria, en vez de reducirse, fue todavía más amplio (3.66\%), con la presentación de dos alianzas electorales -esto último en concordancia con la teoría-.

Tlaxcala (en 2016), Coahuila (en 2016) y el Estado de México (en 2017), por su parte, son estados que de un periodo electoral a otro registraron una disminución en el número de alianzas en contienda cuando, según la teoría, deberían haber registrado un incremento, lo mismo que en su índice de competitividad electoral (Tabla 1).

5. Se obtiene de restar los porcentajes de votación obtenidos por la segunda fuerza política a la vencedora (Ocaña y Oñate, 1999, pp. 236-237). A menor valor del índice, mayor será la competitividad. 
Orlando Espinosa Santiago, Ignacio Daniel

Torres Rodríguez y Ulises Cruz Valencia

Tabla 1. Elecciones por estado con alianzas electorales

en 2010-2016 y 2011-2017

\begin{tabular}{|c|c|c|c|c|c|}
\hline Estado & $A \tilde{n} o$ & $\begin{array}{c}\text { No. de } \\
\text { partidos }\end{array}$ & $\begin{array}{c}\text { No. de } \\
\text { alianzas }\end{array}$ & $\begin{array}{l}\text { No. de } \\
\text { opciones } \\
\text { de voto }\end{array}$ & $\begin{array}{c}\text { Indice de } \\
\text { competitividad } \\
\text { electoral }\end{array}$ \\
\hline \multirow{2}{*}{ Aguascalientes } & 2010 & 6 & 1 & 4 & 5.21 \\
\hline & 2016 & 8 & 1 & 6 & 2.92 \\
\hline \multirow{2}{*}{ Colima } & 2009 & 7 & 3 & 4 & 6.4 \\
\hline & 2016 & 9 & 1 & 6 & 3.7 \\
\hline \multirow{2}{*}{ Chihuahua } & 2010 & 7 & 1 & 4 & 16.36 \\
\hline & 2016 & 9 & 1 & 7 & 8.94 \\
\hline \multirow{2}{*}{ Durango } & 2010 & 8 & 1 & 5 & 1.9 \\
\hline & 2016 & 8 & 2 & 6 & 3.66 \\
\hline \multirow{2}{*}{ Hidalgo } & 2010 & 7 & 2 & 3 & 5.12 \\
\hline & 2016 & 7 & 1 & 5 & 15.26 \\
\hline \multirow{2}{*}{ Oaxaca } & 2010 & 8 & 2 & 4 & 8.21 \\
\hline & 2016 & 10 & 2 & 7 & 7.07 \\
\hline \multirow{2}{*}{ Puebla } & 2010 & 7 & 2 & 3 & 10.3 \\
\hline & 2016 & 10 & 2 & 5 & 11.8 \\
\hline \multirow{2}{*}{ Quintana Roo } & 2010 & 7 & 2 & 3 & 26.23 \\
\hline & 2016 & 8 & 2 & 5 & 8.95 \\
\hline \multirow{2}{*}{ Sinaloa } & 2010 & 6 & 2 & 2 & 5.4 \\
\hline & 2016 & 10 & 2 & 8 & 15.69 \\
\hline \multirow{2}{*}{ Tamaulipas } & 2010 & 7 & 1 & 5 & 30.77 \\
\hline & 2016 & 9 & 1 & 8 & 14.09 \\
\hline \multirow{2}{*}{ Tlaxcala } & 2010 & 9 & 3 & 4 & 7.61 \\
\hline & 2016 & 10 & 1 & 8 & 2.36 \\
\hline \multirow{2}{*}{ Veracruz } & 2010 & 7 & 3 & 3 & 2.55 \\
\hline & 2016 & 11 & 2 & 7 & 4.12 \\
\hline \multirow{2}{*}{ Zacatecas } & 2010 & 7 & 2 & 4 & 19.97 \\
\hline & 2016 & 8 & 2 & 7 & 10.41 \\
\hline \multirow{2}{*}{ Coahuila } & 2011 & 10 & 3 & 4 & 24.92 \\
\hline & 2017 & 14 & 2 & 7 & 2.44 \\
\hline \multirow{2}{*}{ Nayarit } & 2011 & 8 & 2 & 5 & 7.32 \\
\hline & 2017 & 10 & 2 & 8 & 12.27 \\
\hline \multirow{2}{*}{$\begin{array}{l}\text { Estado de } \\
\text { México }\end{array}$} & 2011 & 7 & 2 & 3 & 45.61 \\
\hline & 2017 & 8 & 1 & 6 & 2.69 \\
\hline
\end{tabular}

Fuente: elaboración propia con base en Centro de Investigación para el Desarrollo (2017), información de bases de datos de los institutos electorales de los estados y De la Peña (2017).

\section{6}


Entonces, si la teoría señala lo opuesto, ¿por qué los procesos electorales de estos estados se tornaron más competitivos en ausencia de un mayor número de alianzas?, y ¿qué fue lo que determinó que se presentara una diferencia en términos de la amplia variación del margen de victoria?

Responder a estas preguntas demanda reformular o ampliar la teoría y cambiar de estrategia metodológica para explicar en detalle las paradojas enunciadas. En este sentido, la selección de casos debería enfocarse en cuatro propósitos: el primero sería evidenciar los mecanismos causales que hay detrás de las correlaciones comprobadas por estudios previos en la cuestión; el segundo consistiría en encontrar pautas que expliquen aquellos casos que la teoría no puede; el tercero sería analizar el contraste entre casos en términos de lo previsto por la teoría; y el cuarto sería observar la máxima variación entre casos con escenarios similares (Gerring, 2007 y 2008).

Cabe mencionar que la tradición cualitativa en el estudio de las alianzas electorales no es, en sentido estricto, un ejercicio novedoso, y menos en el caso mexicano a nivel subnacional. Varios de los estudios en la materia son ricos en la descripción del contexto, y detallan los procesos políticos y electorales registrados en la antesala de la formación de una alianza electoral. Además, han utilizado varias técnicas de recolección de datos, como entrevistas a los actores clave de la coyuntura política, análisis de prensa y revisión histórico-política y del desempeño electoral de los partidos (Calvo, 2007; Fernández, 2004; Reynoso, 2011b; Mora, 2011; Reveles, 2011; García, 2017).

No obstante, queda la sensación de que hasta ahora dichos trabajos han dado una explicación limitada porque han dejado fuera elementos sistemáticos encontrados por los estudios previos, omitido los contrastes o controles realizados con otros casos, respaldado argumentos sólo aplicables 
a sus casos de estudio y generado tantas proposiciones que es imposible replicarlos en otros contextos.

Para tratar de solventar algunas de estas limitaciones de la tradición cualitativa en el estudio de las alianzas electorales, el ejercicio propuesto en este artículo sostiene que el fenómeno aliancista contiene elementos sistemáticos -comunes en la aproximación por variables-que perduran en la tradición cualitativa.

En este sentido, los casos forman parte de un conjunto de lugares, espacios o contextos sociales donde se presentan los componentes sistemáticos enunciados por los estudios cuantitativos, pero que adquieren los matices de las condiciones sociopolíticas específicas, las cuales pueden inhibir o fortalecer el efecto esperado de las variables.

En el caso mexicano, también dichas realidades subnacionales pueden indicar aspectos, variables o factores omitidos por la literatura, y en ese sentido, explicar mejor el fenómeno de las alianzas en las elecciones de gobernadores, por ejemplo.

\section{Los estudios cuantitativos sobre la formación de alianzas electorales}

Los estudios cuantitativos son los que concentran la mayor cantidad de trabajos que existen hasta este momento sobre alianzas electorales. Dos enfoques son los más significativos a la hora de enunciar los motivos que dan lugar a la configuración de una alianza electoral: la formación de alianzas en regímenes autoritarios-hegemónicos y el mismo fenómeno en regímenes democráticos.

El primero se enmarca en regímenes donde los partidos de oposición se coaligan con el propósito de obtener algunas prerrogativas o mantener -medianamente- remotas posibilidades de triunfo y fortaleza política ante los embates del régimen y el partido en el Gobierno (Wahman, 2011).

\section{8}


Wahman (2011) encuentra que los partidos son más propensos a formar alianzas cuando perciben más próxima la victoria y cuando logran acordar una agenda conjunta de políticas contraria a la del partido en el Gobierno. También Gandhi y Reuter (2013) ponen en evidencia que la represión electoral combinada con cierta estabilidad de los partidos de oposición favorece la manifestación del fenómeno aliancista.

Los hallazgos reportados por estos trabajos son muy significativos para las investigaciones que versan sobre las elecciones en México, pues si bien la competencia partidista de hace algunos años a la fecha puede considerarse como democrática y competitiva, es innegable que en algunos territorios todavía permea la lógica autoritaria en las prácticas informales y en las relaciones que llevan a la consecución del poder. Estos vestigios que siguen acentuándose en algunos vértices de la arena estatal durante la era democrática son en los que se debe indagar a mayor profundidad.

El segundo enfoque transcurre sobre en un escenario donde las condiciones democráticas de competencia son la regla y no la excepción. Sobre esta línea, pueden encontrarse estudios que atribuyen la explicación de las alianzas electorales a las particularidades institucionales de los regímenes. Aquí, probablemente, los estudios más representativos son los realizados por Golder (2005 y 2006), quien determinó que la desproporcionalidad es factor clave en la conformación de las alianzas, siempre y cuando exista un número elevado de partidos contendiendo y una mayor proximidad ideológica entre ellos.

Otras investigaciones que se desarrollan sobre este enfoque son aquellas que abordan las implicaciones de las alianzas electorales sucedidas en un entorno multinivel y en función de una competitividad de alcance nacional. Machado (2009) da cuenta del fenómeno de verticalización en Brasil, consistente en la obligación de formar la misma coalición en todos los niveles de manera concurrente; Clerici 
(2013) y Clerici y Scherlis (2014) demuestran que los marcos normativos suelen tener un impacto en el balance de poder entre los niveles nacional y subnacional de los partidos, así como sobre su comportamiento aliancista; mientras que Kellam (2015) pone en evidencia que la propensión de los acuerdos preelectorales está en función de los temas de la agenda política y de la ideología.

Por lo que respecta al caso mexicano, resaltan puntualmente los estudios hechos por Miño (2014), quien, como ya se mencionó, observa que el Gobierno dividido vertical está altamente relacionado con la generación de alianzas divergentes, ${ }^{6}$ aunque señala que, cuando existe un Gobierno en dichas condiciones, las probabilidades de formar una alianza divergente aumentan. Esto lo lleva a considerar que los partidos sí consideran la dimensión nacional al momento de conformar una alianza en el ámbito local/estatal.

Devoto y Olmeda (2017) hacen lo propio al demostrar que "los partidos no sólo desarrollan estrategias diferentes entre estados y a lo largo del tiempo, sino, incluso, en diversos distritos en una misma elección y dentro de una misma entidad federativa" (Devoto y Olmeda, 2017, p. 184). Cruz (2019) reconoce esta dinámica aliancista multinivel, y elabora un modelo a partir de la penetración y la difusión territoriales desde la experiencia argentina.

Méndez (2012), por su parte, y después de analizar ciento cinco elecciones -seis legislativas federales, tres presidenciales y noventa y seis para gubernaturas, todo ello en México-, prueba que la normatividad electoral y la

6. Las alianzas divergentes indican la adopción de estrategias distintas en dos o más niveles. En concreto, "Una alianza es divergente cuando dos partidos nacionales rivales, a menudo distantes ideológicamente, se alían en el nivel subnacional con un propósito electoral" (Miño, 20I4, p. 8). No obstante, a partir de la experiencia Argentina, Cruz (2019) llama la atención sobre la dinámica multinivel, se trate o no de alianzas divergentes. De hecho, señala que las coaliciones electorales multinivel son "aquellas que están integradas por varios partidos que disponen de recursos electorales propios para acceder a cargos ejecutivos nacionales y subnacionales en un sistema de partidos multinivel” (Cruz, 2019, pp. I75-I76).

\section{0}


ideología son dos factores que inciden de manera determinante en la formación de las alianzas. Para dicha autora, la competitividad electoral igualmente se presenta como un factor asociado al fenómeno, de tal manera que coincide con Reynoso (2011a) cuando este logra establecer, a través de la prueba de sus hipótesis de señalización y competitividad (Reynoso, 2011a, pp. 13-15), que entre el margen de victoria y la conformación de alianzas electorales existe una asociación.

Cabe mencionar que las alianzas electorales han sido analizadas tanto en sistemas parlamentarios como presidenciales, así como en los tres órdenes de gobierno (federal, estatal y municipal). Ante la evidencia, resulta poco cuestionable el hecho de que el fenómeno aliancista responde primordialmente a una lógica local, aunque es posible distinguir que su configuración se moldea a partir de las dinámicas políticas que se suscitan entre los distintos niveles de gobierno.

\section{La perspectiva cualitativa sobre} la formación de las alianzas electorales

A diferencia de la tradición cuantitativa, el enfoque cualitativo es incipiente en cuanto al análisis de las alianzas electorales. Dos trabajos se han generado al interior de sistemas parlamentarios, y uno en el sistema presidencial. Respecto de los sistemas parlamentarios, el primero de ellos es de Bale, Boston y Church (2006), quienes, al analizar el caso de Nueva Zelanda en 1999, a partir de las trayectorias dependientes o path dependence, distinguieron cómo y por qué los partidos involucrados se asociaron, y que una coalición de Gobierno ${ }^{7}$ será mayormente exitosa si la figura de las alianzas electorales precede la elección, con el argu-

7. Gabinete conformado por miembros de distintos partidos, característicos de los sistemas parlamentarios. 
mento de que dicha convivencia prolongada de las fuerzas políticas favorece su interacción al momento de gobernar.

El segundo trabajo es el de Allern y Aylott (2009), quienes desarrollaron dos estudios de caso - uno en Noruega en 2005 y otro en Suecia en 2006- sobre la primera vez en la que dos o más partidos se unieron en coalición preelectoral. Dado que la formación de estas puntuales alianzas escapaba a las condiciones previstas por los estudios referentes, los autores evidenciaron que los partidos decisivos (o partidos grandes) se coaligan ante un shock externo,${ }^{8}$ priorizan cargos y sacrifican la maximización de votos y su ideología política. A decir de ellos, la inclusión de la investigación histórica para describir y encontrar principios de causalidad en la toma de decisiones de los partidos, basada en un acontecimiento político detonador, se presenta como una herramienta útil para abordar los casos desafiantes.

Finalmente, respecto del sistema presidencial mexicano, la obra coordinada por Reynoso y Espinosa (2017) se enfoca en el subgrupo de las alianzas contra natura o antihegemónicas (PAN-PRD) en elecciones de gobernador. Mediante catorce estudios de caso -once en los que las alianzas se concretaron y tres más en los que no sucedieron-y el análisis comparativo de los veinte casos de dichos tipos de alianzas registrados en elecciones mexicanas hasta 2016, los autores muestran sólida evidencia de que las crecientes expectativas de triunfo en un sistema de hegemonía priista, ${ }^{9}$ en combina-

8. Concepto introducido por Harmel y Janda (1994), entendido como un acontecimiento político sustantivo, ya sea del entorno en general o con relación a los propios partidos políticos, que modifica radicalmente la conducta de estos respecto a las futuras estrategias a implementar para obtener el triunfo.

9. Hallazgo similar al deWahman (20I I), quien analiza el fenómeno de las alianzas en regímenes autoritarios. Esto evidencia que, a pesar de la instauración de prácticas democráticas en un país, si el antes hegemónico partido aún posee cierto poder a nivel subnacional, $y$ dadas las probabilidades extendidas de triunfo de las demás fuerzas políticas, la generación de las alianzas es concretada con el propósito de vencerlo. De alguna manera, guardando toda proporción con respecto a ambos diseños de investigación, el trabajo de Reynoso y Espinosa (2017) ha develado el mecanismo causal en observaciones similares a las asociaciones probabilísticas de 
ción con múltiples y simultáneas elecciones de gobernador que no coincidieron con elecciones presidenciales, ${ }^{10}$ son condiciones que inciden fuertemente en la concreción de este tipo de alianzas. ${ }^{11}$

\section{La necesidad de ampliar la aproximación cualitativa}

De la revisión de la literatura salta a la vista que es menester edificar puentes entre los estudios cuantitativos centrados en variables y la investigación orientada a casos para fortalecer la teoría (Della Porta, 2008, pp. 202-204). La revelación de mecanismos y efectos causales a la luz de los enfoques teóricos de la competitividad electoral apunta a indagar más sobre las conductas y estrategias multinivel de los partidos en contextos dinámicos y cambiantes y sobre los procesos de negociación entre los socios políticos, y, puntualmente para el caso mexicano, a no descartar la lógica y los vestigios autoritarios que aún pueden tener un alto poder explicativo en el plano subnacional, donde se han erigido élites locales que han buscado a toda costa reproducir y mantener la hegemonía política en los estados.

Los estudios de caso se presentan como una opción sumamente factible para ahondar en las explicaciones causales que demanda la complejidad del fenómeno de las alianzas electorales. Poner en práctica la investigación holística, la

Wahman. Sin duda, aplicar esta metodología en algunos de los casos que aborda este autor resultaría sumamente revelador para conocer las diferencias entre las alianzas en contextos autoritarios y las alianzas en contextos postautoritarios. 10. El hecho de que las contiendas para gobernador sean simultáneas en distintos estados, pero asíncronas a la elección presidencial, sugiere que la dimensión multinivel se encuentra presente en la configuración de alianzas a nivel subnacional y que, por ende, los partidos apuestan todo por la elección federal del Ejecutivo. Entre más hay en juego, más mueven sus piezas de forma estratégica e integral. I I. Asimismo, cabe destacar que el estudio consideró una tercera hipótesis: que la fractura de la élite gobernante y los priistas en disponibilidad -aspirantes del PRI que no lograron obtener la candidatura oficial, pero que buscaban obtenerla por otros partidos políticos o alianzas- también tienen incidencia en la conformación de este tipo de alianzas. Sin embargo, esta hipótesis presentó débil evidencia empírica. 
examinación comprensiva de un fenómeno y la recolección de múltiples tipos de evidencias en un contexto "de la vida real" (Gerring, 2007, p. 17), ${ }^{12}$ mediante los estudios de caso, será sumamente clarificador en lo que respecta a las relaciones entre los actores políticos, las particularidades y las tradiciones que subyacen a las prácticas en determinadas regiones del país.

Asimismo, el componente inductivo de la investigación cualitativa permitirá identificar otros factores determinantes previamente obviados o no considerados en las amplias muestras de análisis, además de que mostrará las relaciones políticas y nuevas hipótesis con miras a ser sometidas de manera posterior a muestras más numerosas que permitan generalizarlas empíricamente (Gerring, 2008).

En esa tónica, cabe apuntar que México posee un sistema presidencial que experimentó un alto grado de centralización política. Aun cuando la contienda electoral se reguló y democratizó, todavía pueden constatarse lógicas centralistas de poder en los órdenes locales de Gobierno (estatal y municipal) y marcadas tradiciones partidistas en ciertas entidades del país (Reynoso y Espinosa, 2017), así como mayores o menores vestigios del autoritarismo y clientelismo de antaño.

Por lo anterior, la mayor competitividad electoral no siempre genera la formación de alianzas electorales, y tampoco incrementa en automático esta estrategia aliancista que por muchos años Reynoso (2011a) ha identificado como dominante. En ese tenor, bajo la premisa de aproximarse a los casos de estudio, es necesario formular y proponer un enfoque teórico más profuso para la revisión del fenómeno.

Los partidos políticos en la esfera nacional y subnacional mexicana enfrentan dilemas que tienen que resolver ante la mayor volatilidad del electorado. Si bien es cierto que su

12. Las itálicas son del original. 
principal propósito es acceder a los cargos de representación política, también lo es que buscan otro tipo de beneficios en contextos donde se presentan a competir con escasa presencia electoral. Esa dificultad para crecer en determinadas regiones o contextos los lleva a coaligarse con fuerzas políticas que les otorguen más beneficios que si compitieran individualmente.

Es dable decir que en cada entidad con particularidades y temporalidades distintas hay una potencial distribución de beneficios que se topa con los intereses, situación y comportamiento de cada partido, lo que traslada la discusión sobre la conformación aliancista de un terreno macro de indicadores electorales (Reynoso, 2011a; Miño, 2014) al espacio de negociación e interacción frontal entre partidos donde sus líderes buscan mejorar sus posiciones políticas por medio de la conformación de las alianzas.

Los potenciales socios políticos, ante la incertidumbre de un resultado electoral y la carencia de mecanismos de rendición de cuentas entre ellos, deciden aliarse o no considerando la potencial distribución de los beneficios a partir de los recursos políticos y electorales de que dispongan, así como de sus capacidades y estrategias que les permiten tener una ventaja competitiva al momento de establecer los términos de la coalición.

En este sentido, las históricas condiciones políticas, el momento de la negociación, las arenas de enfrentamiento político, y la distribución de beneficios entre potenciales socios podrían explicar mejor la presencia o ausencia de alianzas electorales en elecciones para gobernador en el caso mexicano.

Para documentar este argumento, entonces, es necesario identificar tanto los lugares donde se ha registrado exitosamente la conformación de las alianzas electorales como aquellos casos donde no se registró el efecto esperado de las variables sistemáticas identificadas por la literatura, lo que 
lleva a la selección de casos y la selección teóricamente guiada de observaciones (Vennesson, 2008; Gerring, 2007 y 2008).

\section{Los casos relevantes para profundizar en el análisis cualitativo de las alianzas electorales}

A partir de una base de datos con el registro de ciento veintiocho elecciones para gobernador en México (19952017), en la que se identificaron casos atípicos en función de la variación porcentual del índice de competitividad electoral, se determinó seleccionar de manera intencionada las observaciones del periodo $2016-2017^{13}$ por tres razones: incluyen el caso con mayor variación en los valores de competitividad electoral (la elección del Estado de México de 2017); la viabilidad en términos de la recolección de datos cualitativos mediante la revisión de prensa y entrevistas a los actores políticos claves para la conformación de las alianzas; y finalmente, porque los dos años del periodo son los de las últimas elecciones estatales de gobernador en que el centro de competencia giraba en torno a la posición dominante del PRI en las entidades federativas.

De dicha selección, se obtuvo que las elecciones para gobernador en México incluyeron un total de dieciséis comicios en el periodo y veinticuatro alianzas electorales (Tabla 1). Lo llamativo de las cifras no es la cantidad de alianzas del periodo, sino que los mismos estados en su elección anterior de gobernador produjeron veintinueve alianzas electorales, lo que indica un ligero descenso, aunque se puede clasificar a dichos estados en aquellos donde se mantienen o incrementan los números de alianzas electorales y aquellos ejemplos donde cae la lógica aliancista. Por otra parte, mientras las opciones de voto registraron un aumento generalizado en

13. Cabe mencionar que la selección intencionada de observaciones se justifica por un interés teórico no exento de riesgos (King, Keohane, y Verba, 2000). A pesar de ello, se reconoce su potencial en la generación de teoría (George y Bennet, 2005). 
las elecciones para gobernador del periodo, el factor de la competitividad electoral se elevó en once de los dieciséis comicios registrados.

De igual manera, se puede presentar la información clasificándola en casos típicos ${ }^{14} \mathrm{y}$ en casos atípicos. ${ }^{15}$ Por ejemplo, según se puede apreciar en la Tabla 2 , siete entidades podrían considerarse como casos típicos y nueve como casos atípicos. La elección de 2016 en Quintana Roo, en ese sentido, podría ser el ejemplo de cómo se eleva el número de alianzas electorales y disminuye el margen de victoria de una elección a otra, en concordancia plena con la teoría. Del grupo de los atípicos, resaltarían particularmente las elecciones en el Estado de México y en Coahuila, pues registran características inesperadas: la cantidad de alianzas disminuye en lugar de incrementarse, mientras el margen de victoria se reduce más del $20 \%$.

Tabla 2. Procesos electorales en México

2016-2017 por tipo de caso y variación

\begin{tabular}{cccc} 
Estado y año & $\begin{array}{c}\text { No. de } \\
\text { alianzas }\end{array}$ & $\begin{array}{c}\text { Dif. de alianzas } \\
\text { respecto al } \\
\text { proceso anterior }\end{array}$ & $\begin{array}{c}\text { Dif. \% indice de } \\
\text { competitividad } \\
\text { electoral con respecto } \\
\text { al proceso anterior }\end{array}$ \\
\hline Quintana Roo (2016) & 2 & Casos típicos & \\
Tamaulipas (2016) & 1 & 0 & $-17.28 \%$ \\
Zacatecas (2016) & 2 & 0 & $-16.68 \%$ \\
Chihuahua (2016) & 1 & 0 & $-9.56 \%$ \\
\hline
\end{tabular}

14. Entendidos como aquellos que reflejan un conjunto esperado de valores con respecto a la comprensión de un fenómeno. Se trata de casos representativos de una relación causal situados en las inmediaciones de la línea de regresión (Gerring, 2008, pp. 648-650).

I5. Entendidos como aquellos casos que muestran valores inusuales con respecto al conocimiento de un tema; son casos pobremente explicados por un modelo. Al no ser representativos, se caracterizan por reflejar residuales altos estadísticamente hablando. Su propósito es ahondar en nuevas explicaciones y factores causales (Gerring, 2008, pp. 655-656). 
Orlando Espinosa Santiago, Ignacio Daniel

Torres Rodríguez y Ulises Cruz Valencia

\begin{tabular}{cccc} 
Estado y año & $\begin{array}{c}\text { No. de } \\
\text { alianzas }\end{array}$ & $\begin{array}{c}\text { Dif. de alianzas } \\
\text { respecto al } \\
\text { proceso anterior }\end{array}$ & $\begin{array}{c}\text { Dif. \% indice de } \\
\text { competitividad } \\
\text { electoral con respecto } \\
\text { al proceso anterior }\end{array}$ \\
\hline Oaxaca (2016) & 2 & 0 & $-1.14 \%$ \\
Veracruz (2016) & 2 & -1 & $1.57 \%$ \\
Hidalgo (2016) & 1 & -1 & $10.14 \%$ \\
Estado de México & & Casos atípicos & \\
(2017) & 1 & -1 & $-42.92 \%$ \\
Coahuila (2017) & 2 & -1 & $-22.48 \%$ \\
Tlaxcala (2016) & 1 & -2 & $-5.25 \%$ \\
Colima (2016) & 1 & 0 & $-2.70 \%$ \\
Aguascalientes (2016) & 1 & 0 & $-2.29 \%$ \\
Puebla (2016) & 2 & 0 & $1.50 \%$ \\
Durango (2016) & 2 & 1 & $1.76 \%$ \\
Nayarit (2017) & 2 & 0 & $4.95 \%$ \\
Sinaloa (2016) & 2 & 0 & $10.29 \%$ \\
\hline
\end{tabular}

Fuente: elaboración propia con base en Centro de Investigación para el Desarrollo (2017) e información de bases de datos de los institutos electorales de los estados.

De manera general, en el plano descriptivo, se podría constatar la presencia de menos alianzas electorales de una elección a otra, lo que se refleja en mayores opciones de voto y en una clara tendencia a favor de la diversificación de la oferta política, caracterizada por nuevos partidos y por nuevas fuerzas políticas independientes, lo que a su vez está dotando de novedosos matices a la competencia electoral en México.

Por ejemplo, la disminución del número de alianzas en contienda, así como la desintegración de las victoriosas, es algo que no está previsto por el conocimiento teórico previo, ya que, de acuerdo con la teoría, las alianzas son generadas en función de replicar y mantener una estrategia para contar con mayores posibilidades de competir y vencer (Reynoso, 2011a). Se carece de estudios que expliquen por qué desa-

\section{8}


parecen o disminuyen las alianzas electorales ante valores constantes de las variables que explican el éxito electoral.

Declarada la selección intencionada de observaciones para el periodo electoral 2016-2017, desde la perspectiva cualitativa, se puede ofrecer un variado repertorio de estudios de caso, dependiendo del propósito final de la investigación. En este artículo, en específico, se revisan opciones disponibles: el caso típico o caso guía, el caso atípico o desviado, el caso típico contra el atípico, y finalmente, la máxima variación entre casos similares, es decir, aquellos casos que consideren la máxima variación de los datos disponibles en las variables.

El ejercicio hipotético muestra los distintos caminos que la investigación cualitativa centrada en uno o pocos casos puede ofrecer al desarrollo de la teoría en ciencias sociales (George y Bennett, 2005) y de las alianzas electorales en particular.

\section{I El caso típico o caso guía}

Bäck y Dumont (2007) señalan que generalmente no hay suficientes investigaciones que den cuenta de los mecanismos causales detrás de los efectos encontrados en los estudios con N grande. Después de identificar los distintos casos en los estudios con muestras grandes, sería posible detectar tanto aquellos que se ajustan perfectamente a los postulados de la teoría como aquellos que se desvían de lo esperado. En este sentido, el caso típico es aquel que, como su nombre lo indica, presenta las características más relevantes y centrales del modelo teórico asumido.

La búsqueda del caso típico suele ser la primera estrategia para la selección de casos porque registra el trazado de $\operatorname{procesos}^{16}$ (process tracing), de manera que se puedan

16. Bennett y Checkel (citados en Bril-Mascarenhas, Maillet, y Mayaux, 2017, p. 664) definen el trazado de procesos como "el análisis de la evidencia sobre los procesos, secuencias y coyunturas de eventos dentro de un caso con el propósito 
identificar los mecanismos causales estándares o ideales, dejando constancia del qué y el cómo; además, suele ser útil para vincular diversos elementos aparentemente aislados sobre el objeto de estudio y, de este modo, observar si los indicadores que se construyeron para la medición de variables fueron adecuados (Vennesson, 2008).

En ocasiones, el conocimiento científico se genera a partir de sólidos estudios de caso, cuyo fin último es generar un conjunto de argumentos o hipótesis que posteriormente podrán refinarse con el avance de las técnicas de recolección de datos y de análisis, para separar los componentes sistemáticos de los aleatorios.

No está por demás enfatizar que en el caso de las alianzas electorales en elecciones de gobernador en México se carece de investigaciones de estudios de caso típico, o bien, de análisis comparativos de pocos casos. Una excepción al respecto es la de Reynoso y Espinosa (2017), pero ellos sólo se enfocan en el estudio de las alianzas antihegemónicas-pragmáticas, que son un subgrupo de las alianzas electorales en general identificadas en el caso mexicano (Reynoso, 2011a).

$\mathrm{Si}$, por ejemplo, se considera exclusivamente el modelo de Reynoso (2011a), el caso de Quintana Roo salta a la vista como el ejemplo más sólido de congruencia con la teoría en el periodo 2016-2017. En la elección de 2010, se había constatado la presencia de dos alianzas y un índice de competitividad electoral de 26.2\%. Para la elección de 2016, se registraron dos alianzas en contienda, el margen de victoria se redujo a $8.95 \%$, y se hizo evidente un incremento en la competitividad electoral de $17.2 \%$. Al observar este caso con detenimiento, es posible distinguir los procesos que sustentan las decisiones de los partidos en el marco de la competitividad, y también cómo interpretan los resultados electorales ante los retos de contiendas futuras.

de desarrollar o probar hipótesis sobre los mecanismos causales que podrían explicar causalmente el caso". 
Los desafíos metodológicos en el estudio de las alianzas electorales subnacionales

\subsection{El caso atípico o desviado}

En contraste con la estrategia anterior, esta se enfoca en elegir aquellos casos que no se ajustan a los supuestos de la teoría, de tal manera que lo que se busca es encontrar explicaciones plausibles para la variación, así como distinguir qué elementos particulares escaparon al análisis cuantitativo o de los estudios disponibles.

$\mathrm{Al}$ revisar las elecciones para gobernador en México en el periodo 2016-2017, el caso atípico más representativo es el del Estado de México. En la elección de 2011, se registraron dos alianzas en la contienda y un índice de competitividad electoral de $45.6 \%$, el histórico más robusto de todos los casos. Si se siguen las premisas de la teoría, la presencia de dos alianzas contrasta con el amplio margen de victoria, aunque también contradice a las expectativas teóricas pues para 2017 únicamente se registró una alianza y la elección fue sumamente competitiva. El margen de victoria de dicho último año fue de $2.69 \% \mathrm{y}$, por tanto, tuvo una variación respecto del año anterior de $42.9 \%$. Esta distancia, sin duda, significa un desafío para las asociaciones estadísticas probadas.

\subsection{El caso típico contra el atípico}

El análisis comparativo puede ser una herramienta útil para analizar dos estudios de caso diferentes en términos de lo presupuesto por la teoría o el resultado de interés con el propósito de encontrar similitudes y tendencias compartidas entre ambos, pero, sobre todo, para identificar las diferencias existentes en el mecanismo causal y en el tipo de elementos que poseen capacidad de configuración sobre el fenómeno, lo que posteriormente sería sometido a validación en otro tipo de casos.

En teoría, el caso típico debería mostrar variación tanto en las variables independientes como en el resultado, y de esta manera, mediante la comparación con el atípico, se 
podrían encontrar dinámicas de configuración de variables distintas tanto en las explicativas como en el resultado.

En nuestro caso de estudio utilizado como ejemplo, en concreto, podrían conducirse los estudios de caso que refirieran a los procesos electorales de Quintana Roo y el Estado de México con miras a profundizar en la causalidad para, mediante un estudio más amplio, determinar qué factores o condiciones se presentan de distinta forma y cómo inciden en la variación.

\subsection{Máxima variación entre casos similares}

Un ejercicio interesante es que después de identificar los casos típicos y atípicos se analice cada conjunto, porque al interior de ellos es probable que estos muestren ser producto de un mecanismo causal con tendencias y elementos similares. En todo caso, resulta más productivo analizar la amplitud máxima de las variables en un conjunto de casos desviados, que son los que escapan a la teoría.

Considerando lo anterior para el ejemplo utilizado en el presente artículo, los casos más desafiantes para las asociaciones validadas en los estudios previos son los de Tlaxcala, Coahuila y el Estado de México.

Los comicios del bienio 2010-2011 registraron tres alianzas electorales y un margen de victoria de $7.61 \%$ en Tlaxcala; tres coaliciones y un margen de victoria de $24.9 \%$ en Coahuila; y dos alianzas y un cómodo índice de competitividad electoral de $45.6 \%$ en el Estado de México. Atípicamente, para el bienio 2016-2017, en los comicios de las tres entidades federativas señaladas se pudo constatar una disminución en el número de coaliciones preelectorales: en Tlaxcala se redujo de tres a una, en Coahuila de tres a dos y en el Estado de México de dos a una.

El cuestionamiento que tensa la teoría pretendería explicar por qué en ausencia de un mayor número de alianzas (asumiendo que estas elevan la competitividad) los índices 
presentaron un súbito reacomodo en los casos estudiados, y pasaron de $7.6 \%$ a $2.3 \%$ en Tlaxcala, de $24.9 \%$ a $2.44 \%$ en Coahuila, ${ }^{17}$ y de $45.6 \%$ a $2.6 \%$ en el Estado de México. En el caso del Estado de México, se constata un vuelco exorbitante en términos de lo planteado por la teoría, además de tratarse de una elección caracterizada por un resultado polémico.

\section{Conclusiones}

Las alianzas electorales han sido escasamente estudiadas a pesar de ser una práctica generalizada alrededor del mundo (Golder, 2005). Una virtud de los estudios al respecto es que por su naturaleza cuantitativa han sido capaces de incluir una amplia cantidad de datos para sustentar sus hipótesis y, con ello, generar solidas afirmaciones sobre el fenómeno aliancista.

La validez externa de las investigaciones cuantitativas se vuelve a confirmar, aunque, como es predecible en cualquier campo de las ciencias sociales, con el tiempo la enorme complejidad de los países y sus divergencias históricas y políticas han puesto en tensión el conocimiento generado cuando se quiere ofrecer una explicación satisfactoria a fenómenos como el aquí analizado.

A pesar del registro de investigaciones cualitativas sobre las alianzas electorales, en el contexto mexicano las indagatorias no suelen seguir el estudio de los casos considerando el conocimiento disponible, controlando las variables, incluyendo los casos negativos, $\mathrm{y}$, sobre todo, identificando

17. El caso de Coahuila es de magnitud más considerable si se toma en cuenta que el balance de las dos alianzas en contienda pudo traducirse en una elección más competida, aunque con las mismas características de los otros dos casos: una reducción en el número de alianzas y un súbito aumento de la competitividad electoral, que lo ubica prácticamente en la media de variación entre los tres. Sin duda, estos son elementos suficientes para conducir un estudio cualitativo para desentrañar sus particularidades. 
lo sistemático de los aspectos aleatorios y circunstanciales propios del lugar de estudio.

Las tensiones ocasionadas por el alcance limitado de las hipótesis dominantes utilizadas para explicar en detalle y satisfactoriamente todos los casos impulsan la necesidad de innovar en las estrategias metodológicas de estudio. Investigar cualitativamente considerando los casos como ejemplos de eventos de un fenómeno específico ofrece amplias oportunidades de investigación, sobre todo cuando se complementa la explicación cuantitativa, se explican mejor las desviaciones de los casos y se aportan -desde una mirada inductiva- nuevas variables que sólo sería posible identificar acudiendo a las condiciones específicas del caso y a los contextos que enmarcan al conjunto. Bajo esta tesitura, los casos típicos, atípicos, de contraste típico-atípico y de máxima variación ofrecen vetas importantes para afinar el conocimiento sobre las alianzas electorales en la esfera subnacional mexicana.

En concordancia con una perspectiva metodológica mixta, el continuointeractivo (Newman y Benz, 1998, pp. 19-26) entre los métodos cuantitativos y cualitativos en cada fase de la investigación (planteamiento del problema, generación de teorías, identificación de variables, definición de argumento-hipótesis, recolección de datos, selección de casos, etc.) ofrece amplias potencialidades para el análisis de los fenómenos políticos. De hecho, algunos estudios recientes sobre alianzas electorales se orientan en esa dirección (Allern y Aylott, 2009; Bale, Boston, y Church, 2006; Bäck y Dumont, 2007).

Reynoso y Espinosa (2017), por su parte, en lugar de considerar la volatilidad y la competitividad electoral como explicativas de las alianzas electorales, sugieren explorar mejor el papel de los partidos políticos, su capacidad de negociación y la distribución de sus recursos, los periodos de su conformación, la historia política local, el calendario 
electoral, la concurrencia de elecciones, los juegos anidados multinivel, las fracturas de las elites, los perfiles de los candidatos aliancistas y el autoritarismo subnacional, entre otros aspectos.

Más allá de la probabilidad de ocurrencia de las alianzas electorales, es necesario saber los porqués, entender mejor los mecanismos causales y encontrar las fallas o condiciones que inhiben el efecto de las variables independientes ampliamente documentadas- sobre la variable dependiente, estando abiertos a la posibilidad de nuevas explicaciones de naturaleza estrictamente inductiva.

Allern, E., y Aylott, N. (2009). Overcoming the fear of comBibliografía mitment: Pre-electoral coalitions in Norway and Sweden. Acta política, 44(3), 259-285. doi: https://doi.org/ 10.1057/ ap.2009.I

Bäck, H., y Dumont, P. (2007). Combining Large-n and SmaII-n Strategies:The Way forward in Coalition Research. West European Politics, 30(3), 467-50I. doi https://doi. org/I0.1080/0I 40238070I 276295

Bale,T., Boston, J., y Church, S. (2006). Natural because it had become just that. Path dependence in pre-electoral pacts and government formation: A New Zealand case study. Australian journal of political science, 40(4), 48I-498. doi https://doi.org//0.1080/1036 I I40500302373

Bril-Mascarenhas, T., Maillet, A., y Mayaux, P. (2017). Process tracing. Inducción, deducción e inferencia causal. Revista de ciencia política,37(3), 659-684. Recuperado de http://www. revistacienciapolitica.cl/index.php/rcp/article/view/354/73

Calvo, R. (2007). Proceso electoral y alternancia en Guerrero. México: Porrúa.

Centro de Investigación para el Desarrollo (2017). Base de datos de resultados electorales 1985-2012 del Centro de Investigación para el Desarrollo, A. C. Recuperado de http:// elecciones.cidac.org/ 
Clerici,P.(2013). Alianzas cruzadas en Argentina. Una aproximación causal desde la teoría. Ciencia política, I 6(julio-diciembre), 8-33. Recuperado de https://revistas.unal.edu. co/index.php/cienciapol/article/view/4 I528/43 I 42

Clerici, P., y Scherlis, G. (20I4). La regulación de las alianzas electorales y sus consecuencias en sistemas políticos multinivel en América Latina. Revista electrónica del Instituto de Investigaciones Ambrosio L. Gioja, 8(I2), 77-98. Recuperado de http://www.derecho.uba.ar/ revistas-digitales/index.php/revista-electronica-gioja/ article/view/77/58

Cruz, F. (2019). Construcción de coaliciones electorales en escenarios desnacionalizados. Un aporte teórico. Revista de estudios políticos, I84(abril-junio), I6I-194. doi https:// doi.org/I 0. I8042/cepc/rep. I84.06

De la Peña, R. (2017). Las elecciones para gobernador en México, 2016: una aproximación comparativa. En R. Valdiviezo, V. Espinosa, y A. Reyes (Coords.), Elecciones bajo nuevas reglas. Cambios y continuidades en los comicios locales. México 2016 (pp. 503-527). México: UAA, SOMEE, BUAP-ICGDE.

Della Porta, D. (2008). Comparative analysis: case oriented versus variable oriented research. En D. Della Porta, y M. Keating, (Eds.), Approaches and methodologies in the social sciences. A pluralist perspective (pp. 198-222). Reino Unido: Cambridge University Press.

Devoto, L.,y Olmeda,J.(20I7). Juntos pero revueltos. Estrategias electorales y coaliciones partidarias para la elección de diputados locales en los estados mexicanos (20002016). Colombia internacional, 90 (abril-junio), I57-I87. doi https://dx.doi.org/10.7440/colombiaint90.2017.06

Espinosa, O. (20I3). Diego Reynoso. La estrategia dominante. Alianzas electorales en los estados mexicanos 1988201 I. Buenos Aires: Flacso Argentina, Teseo, 201 I, pp. 298. Foro internacional, 53(I), 222-226. Recuperado de 
Los desafíos metodológicos en el estudio de las alianzas electorales subnacionales

https://forointernacional.colmex.mx/index.php/fi/article/ Bibliografía view/2 I52/2 | 42

Fearon, J., y Laitin, D. (2008). Integrating Qualitative and Quantitative Methods. En J. Box-Steffensmeier, H. Brady, y D. Collier (Eds.), The Oxford Handbook of Political Methodology (pp. 756-776). Reino Unido: Oxford University Press.

Fernández, R. (2004). Elecciones y alternancia. Guerrero 2005. México: Nuevo Horizonte Editores, Universidad Autónoma de Guerrero.

Gandhi, J., y Reuter, O. (20I3). The Incentives for Pre-electoral Coalitions in Non-democratic Elections. Democratization, 20(I), I37-I59. doi http://dx.doi.org//0.1080/I 3510347.2013 .738865

García, J. (2017). Tamaulipas 1992: la alianza PAN-PRD. En D. Reynoso, y O. Espinosa (Coords.), ¿Alianzas contra natura o antihegemónicas? Las alianzas PAN-PRD en los estados mexicanos (pp. 89-105). México:Tirant Lo Blanch.

George, A., y Bennett, A. (2005). Case Studies and Theory Development in the Social Sciences. Londres: MIT Press.

Gerring,J. (2004).What is a Case Study and What is it Good for? American Political Science Review, 98(2), 34 I-354. doi https://doi.org/I0.1017/S000305540400I I 82

Gerring,J. (2007). Case Study Research. Principles and Practices. Estados Unidos: Cambridge University Press.

Gerring, J. (2008). Case Selection for Case Study Analysis: Qualitative and Quantitative Techniques. En J. Box-Steffensmeier, H. Brady, y D. Collier (Eds.), The Oxford Handbook of Political Methodology (pp. 645-684). Reino Unido: Oxford University Press.

Goertz, G., y Mahoney, J. (2012). A Tale of Two Cultures. Qualitative and Quantitative Research in the Social Sciences. Reino Unido: Princeton University Press.

Golder, S. (2005). Pre-electoral Coalitions in Comparative Perspective: A Test of Existing Hypotheses. Electoral 
Studies, 24,643-663. doi https://doi.org/10.1016/j.electstud.2005.01.007

Golder, S. (2006). Pre-electoral Coalition Formation in Parliamentary Democracies. British Journal of Political Science, 36(2), 193-212. doi https://doi.org/10.1017/ S0007I $23406000 I 23$

González, R. (2017). El exordio de las dinámicas aliancistas: la coalición demócrata potosina (I991). En D. Reynoso, y O. Espinosa (Coords.), ¿Alianzas contra natura o antihegemónicas? Las alianzas PAN-PRD en los estados mexicanos (pp. 67-87). México:Tirant Lo Blanch.

Harmel, R., y Janda, K. (1994). An Integrated Theory of Party Goals and Party Change. Journal of Theoretical Politics, 6(3), 259-287. doi https://doi.org/I0.1 I77/095 I 69289400600300 I

Kellam, M. (2015).Why Pre-Electoral Coalitions in Presidential Systems? British Journal of Political Science, 47(2), 39I4II. doi https://doi.org/I0.1017/S0007 I234I5000 I 98

King, G., Keohane, R., y Verba, S. (2000). El diseño de la investigación social. España:Alianza Editorial.

Machado,A. (2009). Minimum Winning Electoral Coalitions under Presidentialism: Reality or Fiction? The Case of Brazil. Latin American Politics and Society, 5 I (3), 87-I I 0. doi https://doi.org/I0. I I I //j. I548-2456.2009.00057.x Méndez, I. (20I2). Coaliciones preelectorales y competencia partidista en México a nivel federal y local (I994-20 I I). Política y gobierno, I 2(2), I47-I 98. Recuperado de http:// repositorio-digital.cide.edu/handle/ I I65 I/I 622

Miño, J. (20I4). El efecto del Gobierno dividido vertical sobre la formación de alianzas divergentes en los estados mexicanos, 1994-20/3 [tesis de maestría inédita]. Flacso: México. Recuperado de http://flacso.repositorioinstitucional. $\mathrm{mx} / \mathrm{jspui} / \mathrm{handle/}$ I026/70

Mora, S. (20l I). Las alianzas electorales en México: una práctica política. Revista legislativa de estudios sociales y de 
opinión pública, 4(7), 9-3I. Recuperado de http://www3. Bibliografía diputados.gob.mx/camara/00 I_diputados/006_centros_de_estudio/04_centro_de_estudios_sociales_y_ de_opinion_publica/003_accesos_directos/002_publicaciones/003_revista_legislativa

Newman, I.,y Benz, C.R. (1998). Qualitative-Quantitative:Exploring the Interactive Continuum. Estados Unidos: Southern Illinois University Press.

Ocaña, F., y Oñate, P. (1999). Índices e indicadores del sistema electoral y del sistema de partidos. Una propuesta informática para su cálculo. Revista española de investigaciones sociológicas, 86, 223-246. Recuperado de http://www.reis. cis.es/REIS/PDF/REIS_086_II.pdf

Reveles, F. (20I I). El pan y sus alianzas en el 20 I0. El Cotidiano, I65, 6I-7I. Recuperado de http://elcotidianoenlinea. com.mx/pdf/ / 6507.pdf

Reynoso, D. (2010). Alianzas electorales y contingentes legislativos en los estados mexicanos. Revista mexicana de sociología, 72(1), II3-129. Recuperado de http:// revistamexicanadesociologia.unam.mx/index.php/rms/ article/view/I7793/I6970

Reynoso, D. (20I la). Aprendiendo a competir:alianzas electorales y margen de victoria en los estados mexicanos, 1988-2006. Política y gobierno, 18(I), 3-38. Recuperado de http://www.politicaygobierno.cide.edu/index.php/ pyg/article/view/I66/83

Reynoso,V. (20I Ib). Puebla 20 I0: itriunfo de la coalición o fracaso del continuismo? El Cotidiano, 165, 19-29. Recuperado de http://elcotidianoenlinea.com.mx/pdf// 6503. pdf

Reynoso, D.,y Espinosa, O. (Coords.) (2017). ¿Alianzas contra natura 0 antihegemónicas? Las alianzas PAN-PRD en los estados mexicanos. México:Tirant Lo Blanch.

Vennesson, P. (2008). Case studies and process tracing: theories and practices. En D. Della Porta, y M. Keating 
Orlando Espinosa Santiago, Ignacio Daniel

Torres Rodríguez y Ulises Cruz Valencia

Bibliografía

(Eds.), Approaches and methodologies in the social sciences. A pluralist perspective (pp. 223-239). Reino Unido: Cambridge University Press.

Wahman, M.(20I I). Offices and policies -Why do oppositional parties form pre-electoral coalitions in competitive authoritarian regimes? Electoral studies, 30, 642-657. doi https://doi.org/I0.1016/j.electstud.201 I.05.009 